\title{
Etnoecologia de pescadores artesanais sobre espécies nativas e exóticas em
}

\section{reservatórios do semiárido brasileiro}

\author{
Ethnoecology of artisanal fishermen on native and exotic species in brazilian semi-arid reservoirs
}

Etnoecología de los pescadores artesanales sobre las especies nativas y exóticas en embalses semiáridos brasileños

Recebido: 14/01/2022 | Revisado: 22/01/2022 | Aceito: 30/01/2022 | Publicado: 31/01/2022

Leidiane Priscilla de Paiva Batista

ORCID: https://orcid.org/0000-0003-3027-6393 Universidade Federal do Ceará, Brasil E-mail: leidianepriscilla|@gmail.com

Jorge Iván Sanchéz-Botero

ORCID: https://orcid.org/0000-0001-6135-7917 Universidade Federal do Ceará, Brasil

E-mail: jisbar.ufc@gmail.com

Edson Oliveira De Paula

ORCID: https://orcid.org/0000-0003-3134-5012 Universidade Federal do Ceará, Brasil E-mail: edsonoliveirapx@gmail.com

Edson Vicente da Silva

ORCID: https://orcid.org/0000-0001-5688-750X Universidade Federal do Ceará, Brasil

E-mail: cacauceara@gmail.com

\begin{abstract}
Resumo
A etnoecologia busca compreender como diferentes grupos humanos se inter-relacionam com os recursos naturais e ecossistemas que manejam e como os compreendem. Os pescadores passam parte considerável de suas vidas em contato com o ambiente e espécies que manejam, portanto, são portadores de relevantes saberes sobre tais. De modo que compreender as interfaces entre os conhecimentos destas populações e o conhecimento científico, possibilita apontar lacunas a serem investigadas e orientar a conservação dos recursos pesqueiros. Desta forma, objetivou-se comparar os conhecimentos etnoictiológicos dos pescadores artesanais dos açudes públicos Araras e Edson Queiroz (bacia do médio rio Acaraú-CE) com a literatura científica. Para isso, foi selecionada uma população representativa para cada um destes açudes: Ilha de Esaú, para o açude Araras, e Vila São Cosme, para o Edson Queiroz. Foram empregadas estratégias de observação participante, entrevistas semiestruturadas, estímulo visual e turnês-guiadas com pescadores locais. As entrevistas abordaram aspectos etnoecológicos das principais espécies de peixes capturadas na região. Concluiu-se que os pescadores possuem maior conhecimento das espécies nativas, por manejarem-nas há mais tempo e/ou por mudança no comportamento das espécies exóticas ante um ecossistema com características marcadamente divergentes dos ecossistemas de origem destas espécies. Estes conhecimentos podem contribuir para futuros estudos científicos e ser incorporados na elaboração de planos de gerenciamento e manejo sustentável dos recursos hídricos e pesqueiros da região média do Acaraú.
\end{abstract}

Palavras-chave: Conhecimento ecológico local; Pesca artesanal; Etnoictiologia; Ambiente lêntico; Rio Acaraú.

\begin{abstract}
Ethnoecology seeks to understand how different human groups interact with and understand the natural resources and ecosystems they manage. Fishermen spend a considerable part of their lives in contact with the environment and the species they manage, therefore, they are carriers of relevant knowledge about them. Therefore, understanding the interfaces between the knowledge of these populations and scientific knowledge makes it possible to point out gaps to be investigated and to guide the conservation of fishery resources. Thus, the objective was to compare the ethnohistorical knowledge of the artisanal fishermen of the public reservoirs Araras and Edson Queiroz (middle Acaraú River basin-CE) with the scientific literature. For this purpose, a representative population was selected for each of these weirs: Ilha de Esaú, for the Araras weir, and Vila São Cosme, for the Edson Queiroz weir. Participant observation, semi-structured interviews, visual stimulus and guided tours with local fishermen were used. The interviews addressed ethno-ecological aspects of the main fish species caught in the region. It was concluded that fishermen have greater knowledge of native species, because they have managed them for a longer time and/or because of changes in the behavior of exotic species in an ecosystem with markedly divergent characteristics from the ecosystems of origin of these species. This knowledge can contribute to future scientific studies and be incorporated
\end{abstract}


into the development of management plans and sustainable management of water and fish resources in the middle Acaraú region.

Keywords: Local ecological knowledge; Artisanal fishing; Ethnoichthyology; Lentic environment; Acaraú river.

\begin{abstract}
Resumen
La etnoecología trata de entender cómo los distintos grupos humanos interactúan con los recursos naturales y los ecosistemas que manejan y los comprenden. Los pescadores pasan una parte considerable de su vida en contacto con el entorno y las especies que manejan, por lo tanto, son portadores de conocimientos relevantes sobre ellas. La comprensión de las interfaces entre el conocimiento de estas poblaciones y los conocimientos científicos permite señalar las lagunas que hay que investigar y orientar la conservación de los recursos pesqueros. Así, el objetivo es comparar el conocimiento etnoictiológico de los pescadores artesanales de las represas públicas de Araras y Edson Queiroz (Cuenca Media de Acaraú-CE) con la literatura científica. Para ello, se seleccionó una población representativa para cada una de las presas: Ilha de Esaú, para la presa Araras, y Vila São Cosme, para la Edson Queiroz. Se utilizaron estrategias de observación, entrevistas semiestructuradas, estimulación visual y guías con pescadores locales. Las entrevistas abordan aspectos etnoecológicos de las principales especies de peces capturadas en la región. Se concluyó que los pescadores tienen mayor conocimiento de las especies autóctonas, porque las han manejado durante más tiempo y/o por los cambios en el comportamiento de las especies exóticas en un ecosistema con características marcadamente diferentes a los ecosistemas de origen de estas especies. Este conocimiento puede contribuir a futuros estudios científicos y ser incorporado en la elaboración de planes de manejo y manejo sustentable de los recursos hídricos y pesqueros en la región media de Acaraú.
\end{abstract}

Palabras clave: Conocimiento ecológico local; Pesca artesanal; Etnoictiología; Ambiente léntico; Río Acaraú.

\title{
1. Introdução
}

A ecologia é uma ciência que estuda a relação dos seres vivos entre si e destes com o ambiente que habitam. Na língua grega, significa o estudo da casa, incluindo-se os seres que nela residem e os processos funcionais que a tornam habitável (Odum \& Barrett, 2008). Traçando uma perspectiva social, adiciona-se o préfixo etno a palavra ecologia e tem-se o estudo da casa a partir de uma etnia, isto é, de um grupo de pessoa pertencentes a uma mesma cultura. Logo, a etnoecologia, enquanto disciplina, utiliza-se de metodologias elaboradas pela ciência acadêmica (Wieczorkowki et al., 2019) ao mesmo tempo que busca compreender como diferentes grupos humanos se inter-relacionam com os recursos naturais e ecossistemas que manejam e como os compreendem.

O conhecimento ecológico local e o conhecimento científico são formas de saberes diferentes entre si na forma que são construídos e consolidados. Enquanto o primeiro é produzido empiricamente, de modo que se constrói, se mantém e se renova em atividades cotidianas, o segundo é produzido por meio de investigações, experimentos e coletas de dados sistematizados a fim de responder a questionamentos levantados. Ambos têm o potencial de se complementarem por meio de suas convergências e singularidades (Prado \& Murrieta, 2015).

Os pescadores passam parte considerável de suas vidas em contato com o ambiente e espécies que manejam, portanto, são portadores de relevantes saberes sobre tais. Baseados nestes saberes, tomam decisões sobre o uso dos recursos pesqueiros, por conseguinte, são aptos a colaborar com a gestão e o monitoramento dos ecossistemas que atuam. De modo que compreender as interfaces entre os conhecimentos destas populações e o conhecimento científico, possibilita apontar lacunas a serem investigadas e orientar a conservação dos recursos pesqueiros, preferivelmente por meio de coparticipação e diálogo entre a as populações de pescadores envolvidas, academia e gestores.

Historicamente, no semiárido do Nordeste brasileiro, a exploração de peixes sobreviventes em poços fluviais era um dos últimos recursos alimentares durante as secas, exigindo desses grupos conhecimentos sobre o manejo dos peixes. A partir de 1906, foram construídos os primeiros açudes nesta região do Brasil. Aos longos dos anos, várias espécies exóticas de peixes foram introduzidas nos reservatórios nordestinos ao longo das últimas décadas, propositalmente, com o intuito de incrementar a produtividade pesqueira, e acidentalmente, por escape da aquicultura para o ambiente (Gubiani, 2018).

Ante a necessidade de manejo das espécies nativas e introduzidas por parte dos pescadores artesanais desses ecossistemas, o presente estudo objetivou comparar os conhecimentos etnoictiológicos de pescadores artesanais de 
reservatórios nordestinos com a literatura científica. Partiu-se da hipótese que os pescadores artesanais de reservatórios nordestinos possuem conhecimentos etnoecológicos sobre as espécies exóticas de peixes capturadas que contrastam com as informações disponíveis na literatura científica, enquanto os conhecimentos acerca das espécies nativas estão de acordo com a bibliografia acadêmica. Isto porque os pescadores manejam as espécies nativas há mais tempo do que as introduzidas. Desta forma, esperou-se que os pescadores conhecessem melhor as espécies nativas do que as espécies exóticas.

Faz-se necessário salientar que comparar os conhecimentos tradicionais aos científicos não tem o intuito de validação, adotando como referencial a ciência como portadora de saber verdadeiro e padrão. O que se pretendeu neste estudo foi possibilitar um diálogo entre visões de mundo diferentes, evitando-se um centrismo, seja em favor das comunidades locais ou da academia.

\section{Metodologia}

As comunidades de pescadores artesanais Ilha de Esaú e Vila São Cosme estão localizadas, respectivamente, no município de Hidrolândia (às margens do reservatório Paulo Sarasate, mais conhecido por Araras) e no município de Santa Quitéria (que abriga o açude Edson Queiroz, localmente chamado de Serrote). Estes reservatórios compõem o médio curso da bacia hidrográfica do rio Acaraú, situados no noroeste do estado do Ceará, Brasil. O açude Araras foi construído entre os anos de 1951 e 1958, e possui área de $3.520 \mathrm{~km}^{2}$. Por sua vez, o açude Serrote foi construído entre 1984 e 1987, e possui área de $1.765 \mathrm{~km}^{2}$.

As duas comunidades foram selecionadas com base nos critérios: predominância da pesca artesanal como atividade econômica realizada pelos moradores; número de habitantes; acessibilidade por via terrestre; tempo de existência da localidade, com preferência para as mais antigas na região. Com base em informações do Departamento Nacional de Obras Contra a Seca (DNOCS) e dos moradores de cada localidade, estimou-se em sessenta pescadores artesanais ativos na Ilha de Esaú e cinquenta na Vila São Cosme, com população respectiva de 270 e 225 pessoas. As duas localidades existem desde a inauguração dos açudes a maioria dos habitantes tem a pesca como principal atividade econômica, havendo, em menor escala, a realização de agricultura familiar e trabalhos informais para o complemento da renda. Os pescadores utilizam em sua totalidade pequenas embarcações movidas a remo. Os principais apetrechos de pesca foram rede de espera, anzóis e tarrafas.

Posteriormente, foram feitas visitas às comunidades selecionadas para realização de entrevistas parcialmente estruturadas. A princípio, buscou-se investigar o cotidiano comunitário por meio do convívio com membros das localidades selecionadas para o estudo, por meio de estadias com durações de até uma semana na casa de moradores das localidades, participação em reuniões e rodas de conversas com os pescadores, acompanhamento em trajeto pelo açude para retirada de redes de espera, participação em atividades de limpeza do pescado.

Por sua vez, a entrevista parcialmente estruturada foi composta por tópicos fixos e tópicos adaptados ao longo da pesquisa, com a finalidade de direcionar o diálogo para o objeto pesquisado (os saberes etnoictiológicos dos pescadores), permitindo a emersão de novos temas ao longo da entrevista (Albuquerque et al., 2010). Nesta etapa, foram abordados aspectos gerais da pesca, assim como, da ecologia trófica e reprodutiva das principais etnoespécies de peixes capturados pelos pescadores nos reservatórios. Os entrevistados foram selecionados aleatoriamente, não havendo distinção entre sexo, idade ou qualquer outro fator. O termo de consentimento de livre esclarecido foi feito oralmente. Entrevistou-se vinte pescadores na Vila São Cosme e vinte e quatro na Ilha de Esaú. Os registros foram feitos em diário de campo e em gravações de áudio (quando autorizadas pelos entrevistados).

As principais etnoespécies foram identificadas em conversas com os moradores, baseando-se na abundância e no valor econômico. Assim, foram selecionadas três etnoespécies nativas e três exóticas à bacia hidrográfica do rio Acaraú, respectivamente: curimatã (Prochilodus brevis Steindachner, 1874), piau (Leporinus spp.) e traíra (Hoplias spp.); tilápia 
(Oreochromis niloticus Linnaeus, 1758), pescada (Plagioscion squamosissimus Heckel, 1840) e tucunaré (Cichla monoculus Spix \& Agassiz, 1831).

As espécies foram confirmadas junto ao Laboratório de Ecologia Aquática da Universidade Federal do Ceará, por meio do projeto "Produtividade pesqueira em açudes da bacia do rio Acaraú, estado do Ceará, Brasil", do qual o presente estudo fez parte. Este projeto realizou coletas experimentais de peixes nos dois açudes estudados, assim, como a identificação taxonômica dos exemplares coletados. As coletas de material biológico, de informação socioeconômicas e etnoecológicas nos reservatórios Araras e Edson Queiroz foram licenciadas pelo IBAMA.

Este estudo considerou etnoespécies os táxons reconhecidos localmente que possuem correspondência com uma ou mais espécies lineanas presentes na ictiologia científica. A comparação dos conhecimentos etnoictiológicos dos pescadores com a literatura científica ocorreu através de levantamento bibliográfico em livros, publicações acadêmicas, e bases de dados sobre a ecologia e taxonomia das espécies selecionadas.

Construiu-se uma matriz de dados binários com as respostas apresentadas pelos pescadores e as respostas encontradas na literatura científica. Logo, para cada açude, pescador e etnoespécie, avaliou-se cada um dos seguintes aspectos relacionados à ecologia dos peixes: origem de ocorrência quanto à bacia do rio Acaraú, agrupamento (formação de cardume), tipo, local e horário de alimentação, período e local de desova, e cuidado parental.

Usou-se análise de variância (ANOVA) para a comparação das respostas, com o intuito de apresentar a relação entre o conhecimento científico e os saberes dos pescadores estudados acerca de cada uma das espécies selecionadas. Foi utilizada também a análise de frequência, através do programa estatístico Past (PAST, 2001). A ANOVA avaliou a similaridade entre as médias de respostas para as etnoespécies em cada açude. Com a hipótese nula estatística de igualdade entre todas as médias. Com o resultado da ANOVA, utilizou p-valor inferior ao nível de significância 0,05 para rejeição da hipótese nula, indicando diferença entre as médias referentes às etnoespécies. O nível de confiança foi de 95\%. Para a análise de frequência, adotou-se o percentual igual ou menor a $10 \%$ como indicativo de significativa divergência entre as respostas fornecidas pelos pescadores e os dados científicos.

\section{Resultados e Discussão}

Pescadores artesanais de várias partes do Brasil demonstraram-se portadores de amplos conhecimentos acerca de biologia, ecologia e comportamento das espécies de peixes que manejam (Ladislau et al., 2021; Begossi et al., 2016). Pesquisas sobre estes conhecimentos tem o potencial de contribuir com a elaboração de planos manejo sustentável e com a conservação dos recursos pesqueiros.

Nos açudes públicos Edson Queiroz e Araras, os pescadores descreveram conhecimentos ecológicos sobre aspectos gerais, tróficos e reprodutivos dos principais peixes capturados. A seguir, realizou-se uma comparação entre duas linguagens diferentes: a científica e a tradicional, a respeito dos saberes relatados pelos pescadores. Adotou-se o conhecimento disponível na literatura científica como ponto de partida, considerando-se as informações sobre aspectos bioecológicos dos principais peixes comerciais (Tabela 1) da região estudada: 
Tabela 1. Aspectos biológicos e ecológicos das principais espécies de peixes comerciais capturadas pelos pescadores nos açudes públicos Araras e Edson Queiroz, bacia do rio Acaraú Ceará.

\begin{tabular}{|c|c|c|c|c|c|c|c|c|}
\hline Espécie de peixes & Origem & $\begin{array}{l}\text { Habito } \\
\text { alimentar }\end{array}$ & $\begin{array}{c}\text { Zona de } \\
\text { alimentação }\end{array}$ & $\begin{array}{c}\text { Período } \\
\text { alimentar }\end{array}$ & $\begin{array}{l}\text { Período de } \\
\text { desova }\end{array}$ & $\begin{array}{c}\text { Local de } \\
\text { desova }\end{array}$ & Agrupamento & $\begin{array}{l}\text { Cuidado } \\
\text { Parental }\end{array}$ \\
\hline $\begin{array}{c}\text { Curimatã } \\
\text { Prochilodus brevis }\end{array}$ & Nativa $^{1}$ & Detritívoro $^{2}$ & Filhote: litoral $^{3}$ & Diurno $^{4}$ & Chuvoso $^{5}$ & $\begin{array}{l}\text { Ambiente } \\
\text { lótico }^{5}\end{array}$ & Reprodutivo $^{6}$ & Ausente $^{6}$ \\
\hline $\begin{array}{c}\text { Piau } \\
\text { Leporinus spp. }\end{array}$ & Nativa $^{1}$ & Onívoro 7 & Limnética $^{7}$ & Diurno $^{7}$ & Chuvoso $^{6}$ & $\begin{array}{l}\text { Ambiente } \\
\text { lótico }^{5}\end{array}$ & Reprodutivo $^{5}$ & Ausente ${ }^{5}$ \\
\hline $\begin{array}{c}\text { Traíra } \\
\text { Hoplias spp. }\end{array}$ & Nativa $^{1}$ & $\begin{array}{c}\text { Carnívoro } \\
\text { ou piscívoro }^{8}\end{array}$ & Litoral $^{9}$ & Noturno 9 & Chuvoso $^{10}$ & Litoral $^{10}$ & Estágio juvenil ${ }^{5}$ & Presente $^{11}$ \\
\hline $\begin{array}{l}\text { Cará-tilápia } \\
\text { Oreochromis } \\
\text { niloticus }\end{array}$ & Exótica $^{1}$ & Onívoro ${ }^{12}$ & $\begin{array}{c}\text { Juvenil: litoral; } \\
\text { Adulto: limnética }{ }^{12}\end{array}$ & Diurno $^{13}$ & Anual $^{14}$ & Litoral $^{14}$ & Reprodutivo $^{14}$ & Presente $^{14}$ \\
\hline $\begin{array}{c}\text { Pescada } \\
\text { Plagioscion } \\
\text { squamosissimus }\end{array}$ & Exótica $^{1}$ & Carnívoro $^{15}$ & Limnética $^{16}$ & $\begin{array}{c}\text { Crepuscular e } \\
\text { noturno }^{17}\end{array}$ & Anual $^{18}$ & $\begin{array}{l}\text { Ambiente } \\
\text { lótico }^{19}\end{array}$ & Todos os estágios ${ }^{20}$ & Ausente $^{20}$ \\
\hline $\begin{array}{c}\text { Tucunaré } \\
\text { Cichla monoculus }\end{array}$ & Exótica $^{1}$ & $\begin{array}{l}\text { Carnívoro }{ }^{21} \text { ou } \\
\text { piscívoro }^{5}\end{array}$ & $\begin{array}{l}\text { Filhote: litoral; } \\
\text { Adulto: limnética }\end{array}$ & $\begin{array}{c}\text { Matutino e } \\
\text { crepuscular }^{22}\end{array}$ & Chuvoso $^{23}$ & Litoral $^{23}$ & Estágio juvenil ${ }^{23}$ & Presente $^{23}$ \\
\hline
\end{tabular}

Literatura Utilizada: 1. Froese \& Pauly, (2021) / 2. Moura et al., 2018/3. Resende, (1992) / 4. Fugi et al., (1996) / 5. Lowe-Mcconnell, (1999) / 6. Winemiller, (1989) / 7. Durães et al., (2001) / 8. Pompeu \& Godinho, (2001) / 9. Casatti et al., 2001)/ 10. Marques et al., 2001) / 11. Garcia et al., (2019) / 12. Bwanika et al., (2006) / 13. Pratiwy \& Kohbara, (2018) / 14. Philippart \& Ruwet, (1982) / 15. Costa \& Angelini, (2020) / 16. Goulding, (1980) / 17. Hahn et al., (1999) / 18. Camargo \& Lima-Júnior, (2008) / 19. Capra \& Bennemann, (2009) / 20. Borie et al., (2014) / 21. Novaes et al., (2004) / 22. Rabelo \& Araujo-Lima, (2002) / 23. Winemiller, (2001). Fonte: Elaborada pelos autores.

\subsection{Aspectos Gerais de Etnoecologia}

Considerando-se os aspectos gerais da ecologia dos peixes segundo os pescadores da Vila São Cosme e da Ilha de Esaú, expõe-se a seguir o resultado da ANOVA (Tabela 2), que relacionou a resposta dos pescadores aos dados presentes na literatura científica para origem (com relação a bacia hidrográfica do rio Acaraú) e agrupamento das principais etnoespécies de peixes capturadas na região.

Tabela 2. Resultado da ANOVA para aspectos gerais da ecologia dos principais peixes capturados pelos pescadores da Vila São Cosme e Ilha de Esaú. Em negrito, p-valor inferior ao nível de significância 0,05. GL: grau de liberdade; V: Vila São Cosme; I: Ilha de Esaú.

\begin{tabular}{ccccccccccc}
\hline \multirow{2}{*}{$\begin{array}{c}\text { ASPECTOS } \\
\text { GERAIS }\end{array}$} & \multicolumn{2}{c}{ SOMA DOS } & \multicolumn{2}{c}{ GL } & \multicolumn{2}{c}{ MÉDIA DOS QUADRADOS } & \multicolumn{2}{c}{ RAZÃO-F } & \multicolumn{2}{c}{ P-VALOR } \\
& QUADRADOS & & & & & & & & \\
& V & I & V & I & V & I & V & I & V & I \\
\hline Origem & 0,04261 & 12,410 & \multirow{2}{*}{1} & 0,04261 & 12,4103 & 0,01 & 4,32 & 0,905 & $\mathbf{0 , 0 3 9}$ \\
Agrupamento & 0,47619 & 0,0581 & & & 0,47619 & 0,05806 & 0,16 & 0,02 & 0,689 & 0,889 \\
\hline
\end{tabular}

Fonte: Elaborada pelos autores.

Ao examinar a ANOVA, somente o item origem para a Ilha de Esaú obteve p-valor inferior ao nível de significância 0,05. Este valor induz a rejeição da hipótese nula, indicando que pelo menos umas das médias diferiram das demais, no que se refere a origem das etnoespécies. Com isso, considerou-se a análise de frequência (Tabela 3) para averiguar quais espécies os pescadores apresentaram dados diferentes aos da literatura científica, considerando-se o percentual menor ou igual a 10\% para apontar considerável divergência entre os aspectos gerais de ecologia dos peixes. 
Tabela 3. Análise de frequência das respostas condizentes com a literatura científica sobre aspectos gerais da ecologia dos principais peixes capturados pelos pescadores da Vila São Cosme e Ilha de Esaú. Em negrito, frequência menor ou igual a 10\% que indica divergência entre respostas científicas e dos pescadores. V: Vila São Cosme; I: Ilha de Esaú.

\begin{tabular}{|c|c|c|c|c|}
\hline \multirow{3}{*}{ ETNOESPÉCIE } & \multicolumn{4}{|c|}{ ASPECTOS GERAIS } \\
\hline & \multicolumn{2}{|c|}{ Origem } & \multicolumn{2}{|c|}{ Agrupamento } \\
\hline & $\mathbf{V}$ & $\mathbf{I}$ & $\mathbf{V}$ & $\mathbf{I}$ \\
\hline Curimatã & $16,67 \%$ & $16,67 \%$ & $15,83 \%$ & $15,97 \%$ \\
\hline Piau & $16,67 \%$ & $16,67 \%$ & $15,83 \%$ & $15,28 \%$ \\
\hline Traíra & $16,67 \%$ & $15,97 \%$ & $12,50 \%$ & $11,81 \%$ \\
\hline Cará-tilápia & $10 \%$ & $11,81 \%$ & $14,17 \%$ & $12,50 \%$ \\
\hline Pescada & $\mathbf{5 , 8 3 \%}$ & $5,56 \%$ & $14,17 \%$ & $15,28 \%$ \\
\hline Tucunaré & $7,50 \%$ & $14,58 \%$ & $15 \%$ & $15,28 \%$ \\
\hline
\end{tabular}

Fonte: Elaborada pelos autores.

Com os dados de frequência para origem das etnoespécies, quanto à bacia hidrográfica do rio Acaraú, adotando-se o valor menor ou igual a 10\% como indicativo de contraste com a literatura, observou-se que os pescadores da Vila São Cosme consideraram que as espécies exóticas $O$. niloticus (cará-tilápia), P. squamosissimus (pescada) e C. monoculus (tucunaré) são nativas à bacia hidrográfica do rio Acaraú. Porém, entre estas, o P. squamosissimus foi o que mostrou percentual mais baixo tanto para a Ilha de Esaú quanto para a Vila São Cosme. Ainda, segundo o resultado da ANOVA, as diferenças entre médias de origens da etnoespécies na Ilha de Esaú não foram significativas, uma vez que p-valor foi superior ao nível de significância 0,05 .

Os pescadores das duas localidades manejam estes peixes cotidianamente, uma vez que se tratam de espécies importantes para a economia local. Possivelmente por este fato, os pescadores da Vila São Cosme consideraram todas as espécies exóticas como nativas, e os pescadores da Ilha de Esaú acreditaram que a pescada é nativa à região. Dada a elevada frequência de manuseio destas, efetuou-se um grau de familiaridade, que, ante a relação pescador-peixe, estes trabalhadores não identificam etnoespécies de outra origem que não seja a bacia do rio Acaraú.

No que se refere ao agrupamento pelas espécies, os pescadores dos dois açudes e a literatura científica apresentaram informações condizentes, tanto para as espécies nativas, quanto para as espécies exóticas. Este dado revela que os pescadores das localidades em estudo confirmaram empiricamente os conhecimentos produzidos pela academia. Tais experiências deramse, basicamente, pela forma como os pescadores utilizam-se de apetrechos multiespecíficos, como a rede-de-espera, para captura destes peixes. Durante este processo, eles observam sobre o agrupamento ao apanharem os peixes individualmente ou em cardumes, nomeados localmente por peixes de rebanho. Notou-se, assim, que os saberes etnoictiológicos, para esta característica, são obtidos durante atividades cotidianas (Pereira \& Diegues, 2010), através da apropriação da natureza e da reprodução exaustiva de determinada experiência.

\subsection{Etnoecologia Trófica}

Quanto à ecologia trófica, os tópicos analisados são: tipo, local e horário de alimentação. Como apresentado na tabela 1, o tipo de alimentação foi classificado, de acordo com a espécie, em piscívoro, carnívoro, onívoro ou detritívoro. Quanto ao local de alimentação, as categorias consideradas foram área litorânea e zona limnética. O horário de alimentação foi distribuído ao longo do dia, em hábitos crepuscular, noturno e/ou diurno. Embora utilizando-se de termos locais, as respostas dos pescadores, foram correspondentes a estas categorias. 
Comparando-se a Tabela 4 com os resultados da ANOVA a respeito da ecologia trófica dos peixes em estudo, através do p-valor, a hipótese nula foi rejeitada para tipo e horário de alimentação em ambas as localidades em estudo, insinuando que a diferença entre as médias de respostas para as etnoespécies possuem diferença significativa.

Tabela 4. Resultado da ANOVA para ecologia trófica dos principais peixes capturados pelos pescadores da Vila São Cosme e Ilha de Esaú. Em negrito, p-valor inferior ao nível de significância 0,05. GL: grau de liberdade; V: Vila São Cosme; I: Ilha de Esaú.

\begin{tabular}{ccccccccccc}
\hline \multirow{2}{*}{$\begin{array}{c}\text { ASPECTOS } \\
\text { TRÓFICOS }\end{array}$} & \multicolumn{2}{c}{ SOMA DOS } & \multicolumn{2}{c}{ GL } & \multicolumn{2}{c}{ MÉDIA DOS } & \multicolumn{2}{c}{ RAZÃO-F } & \multicolumn{2}{c}{ P-VALOR } \\
& V & I & V & I & V & I & V & I & V & I \\
\hline Tipo & 37,6252 & 16,5069 & & 37,6252 & 16,5069 & 14,21 & 5,81 & $\mathbf{0 , 0 0 0 3}$ & $\mathbf{0 , 0 1 7 2}$ \\
Local & 0,0379 & 3,3088 & \multirow{2}{*}{1} & 0,0379 & 3,3088 & 0,01 & 1,13 & 0,9102 & 0,2901 \\
Horário & 38,1349 & 25,9824 & & 38,1349 & 25,9824 & 14,43 & 9,36 & $\mathbf{0 , 0 0 0 2}$ & $\mathbf{0 , 0 0 2 6}$ \\
\hline
\end{tabular}

Fonte: Elaborada pelos autores.

Do mesmo modo, foi necessário observar o resultado da análise de frequência (Tabela 5) para saber quais etnoespécies induziram o p-valor menor que 0,05 . Com isso, notou-se que, para tipo de alimentação, o cará-tilápia $(O$. niloticus) entre os pescadores da Vila São Cosme, e o piau (Leporinus spp.) entre os pescadores da Ilha de Esaú obtiveram menos que $10 \%$ de frequência de resposta semelhantes a literatura científica.

Tabela 5. Análise de frequência das respostas condizentes com a literatura científica sobre ecologia trófica dos principais peixes capturados pelos pescadores da Vila São Cosme e Ilha de Esaú. Em negrito, frequência menor ou igual a 10\% que indica divergência entre respostas científicas e dos pescadores. V: Vila São Cosme; I: Ilha de Esaú.

\begin{tabular}{|c|c|c|c|c|c|c|}
\hline \multirow{3}{*}{ ETNOESPÉCIE } & \multicolumn{6}{|c|}{ ALIMENTAÇÃO } \\
\hline & \multicolumn{2}{|c|}{ Tipo } & \multicolumn{2}{|c|}{ Local } & \multicolumn{2}{|c|}{ Horário } \\
\hline & $\mathbf{V}$ & I & $\mathbf{v}$ & I & $\mathbf{V}$ & I \\
\hline Curimatã & $15 \%$ & $15,97 \%$ & $15 \%$ & $15,28 \%$ & $10 \%$ & $7,64 \%$ \\
\hline Piau & $10,83 \%$ & $\mathbf{9 , 7 2 \%}$ & $15,83 \%$ & $15,97 \%$ & $10 \%$ & $9,03 \%$ \\
\hline Traíra & $15,83 \%$ & $16,67 \%$ & $15 \%$ & $15,28 \%$ & $13,33 \%$ & $13,89 \%$ \\
\hline Cará-tilápia & $7,50 \%$ & $11,11 \%$ & $15,83 \%$ & $15,28 \%$ & $6,67 \%$ & $9,03 \%$ \\
\hline Pescada & $15,83 \%$ & $16,67 \%$ & $16,67 \%$ & $15,97 \%$ & $14,17 \%$ & $6,94 \%$ \\
\hline Tucunaré & $16,67 \%$ & $16,67 \%$ & $15,83 \%$ & $16,67 \%$ & $15,83 \%$ & $13,19 \%$ \\
\hline
\end{tabular}

Fonte: Elaborada pelos autores.

Este resultado contrastante deu-se, principalmente, por estas espécies possuírem hábitos alimentares onívoros (Bwanika et al., 2006; Durães et al., 2001), o que pode ter induzido os pescadores a simplificarem a resposta, citando apenas um tipo de alimento. Logo, repetidamente, os pescadores referiram-se a elas como "comedoras de lama", aludindo ao hábito detritívoro.

Outra explicação admissível, que, porém, demanda um estudo ecológico destas espécies, fundamenta-se na disponibilidade de alimento, pois na insuficiência de alimento preferencial, os peixes procuram itens menos preferenciais em uma tendência adaptativa com fins de prevenir desperdício energético na busca de alimentos escassos (Dill, 1983). Nestes termos, levanta-se a hipótese de que a introdução nos açudes das espécies predadoras $P$. squamosissimus e $C$. monoculus, 
possa ter causado alteração na cadeia trófica, acarretando mudança de hábitos alimentares nas espécies onívoras Leporinus spp. e $O$. niloticus, levando estas a se alimentarem, preferencialmente, de detritos, na escassez de outros tipos de alimentos, como o camarão (Macrobrachium sp.).

A observação do conteúdo estomacal dos exemplares capturados, permitiu aos pescadores conhecer a respeito do tipo de alimentação dos peixes. Esta técnica é utilizada também na academia como forma de analisar a ecologia trófica da fauna íctica (Gonçalves et al., 2018). Esta sobreposição de técnicas entre os pescadores e a ciência demonstra que ambas, por meio de observações metódicas, chegaram a mesma conclusão quanto a forma de conhecer o hábito alimentar dos peixes. Deste modo, o conhecimento elaborado popularmente também é ciência.

Com relação ao horário alimentar dos peixes, entre os pescadores da Vila São Cosme e da Ilha de Esaú, as espécies nativas curimatã ( $P$. brevis) e piau (Leporinus spp.) e as espécies exóticas cará-tilápia $(O$. niloticus) e pescada $(P$. squamosissimus), esta última somente na Ilha de Esaú, obtiveram frequência de respostas menor ou igual a $10 \%$ condizente com a literatura.

Por conseguinte, a elevada quantidade de respostas contrastantes com as informações disponíveis na literatura científica deu-se pelo fato dos pescadores destas localidades, frequentemente, relacionarem o horário de alimentação de cada etnoespécie com o horário que eles a capturam, pois acreditam que este é o momento mais provável de encontrarem o peixe, pois nesta ocasião, o animal deixa o seu refúgio e desloca-se pelo açude em buscar de alimento. Entretanto, como a principal armadilha utilizada por eles é a rede de espera, ou seja, uma armadilha que não utiliza isca, a correlação feita entre horário de captura e horário de alimentação do peixe nem sempre foi aplicável.

Considerando-se o local de alimentação, é válido destacar que, todas as espécies conseguiram percentual acima de 10\%. Isto demonstra que os pescadores da Ilha de Esaú e da Vila São Cosme vivenciaram as informações obtidas pela literatura científica para todas as espécies salientadas neste estudo. Diante disso, estes pescadores apresentaram sólido conhecimento a respeito do habitat trófico das espécies capturadas em açudes do alto rio Acaraú.

Conhecer a respeito do local de alimentação de uma espécie de peixe é uma informação valiosa para o sucesso pesqueiro, pois, ainda que os peixes não sejam capturados nos seus horários de alimentação, este local tende a ser próximo ao ambiente de refúgio, posto que, geralmente, os indivíduos de uma espécie evitam percorrer longas distâncias em busca de alimentos (Abelha et al., 2001). Este fato é mais comumente observado quando o habitat do peixe é localizado próximo às macrófitas aquáticas em um ecossistema lêntico, visto que, este tipo de flora funciona como local de alimentação, desova, desenvolvimento e refúgio (Sánchez-Botero \& Araujo-Lima, 2001). De acordo com a literatura, as espécies referentes a quatro das seis etnoespécies abordadas neste estudo (cará-tilápia, curimatã, tucunaré e traíra) alimentam-se na margem do açude próximo à vegetação (Bwanika et al., 2006; Resende, 1992; Lowe-Mcconnell, 1999; Casatti et al., 2001).

Segundo Marques et al. (2001), conhecer profundamente a ecologia trófica da ictiofauna é um elemento útil para a atividade de pesca. Este raciocínio foi comprovado entre os pescadores da Vila São Cosme e da Ilha de Esaú, que demonstraram conhecimento detalhado acerca da ecologia trófica dos peixes capturados e convergem com a literatura científica, principalmente, no concernente aos hábitos relacionados ao tipo e ao local de alimentação. Logo, a construção e a transmissão de conhecimentos advêm do apego ao uso contínuo de recursos (Diegues, 2014), onde não há diferenciação entre o ato conviver-trabalhar e o ato de ensinar-aprender.

\subsection{Etnoecologia Reprodutiva dos Peixes}

No que concerne à ecologia reprodutiva, considerou-se período, local de desova e cuidado parental das espécies ictiológicas em análise. Com isso, de acordo com a tabela de informações bioecológicas (Tabela 1), o período de desova pode ser chuvoso ou em todos os meses do ano a depender da espécie considerada. O local de desova apresentou-se na área litorânea 
ou em ambientes lóticos. O cuidado parental foi definido em ausente ou presente. As respostas, com os termos locais utilizados pelos pescadores, foram ajustadas nestas categorias.

O resultado da ANOVA (Tabela 6) sobre os aspectos reprodutivos das principais espécies de peixes para os açudes Araras e Edson Queiroz levou à rejeição da hipótese nula para período de desova, em ambas as localidades, e para cuidado parental na Vila São Cosme. Isto indica divergência pelo menos em uma das médias das etnoespécies com relação às demais. Assim, a análise de frequência apontará onde está a diferença.

Tabela 6. Resultado da ANOVA para ecologia reprodutiva dos principais peixes capturados pelos pescadores da Vila São Cosme e Ilha de Esaú. Em negrito, p-valor inferior ao nível de significância 0,05. GL: grau de liberdade; V: Vila São Cosme; I: Ilha de Esaú.

\begin{tabular}{|c|c|c|c|c|c|c|c|c|c|c|}
\hline \multirow{2}{*}{$\begin{array}{c}\text { ASPECTOS } \\
\text { REPRODUTIVOS }\end{array}$} & \multicolumn{2}{|c|}{$\begin{array}{c}\text { SOMA DOS } \\
\text { QUADRADOS }\end{array}$} & \multicolumn{2}{|c|}{ GL } & \multicolumn{2}{|c|}{ MÉDIA DOS QUADRADOS } & \multicolumn{2}{|c|}{ RAZÃO-F } & \multicolumn{2}{|c|}{ P-VALOR } \\
\hline & $\mathbf{V}$ & $\mathbf{I}$ & $\mathbf{V}$ & I & $\mathbf{v}$ & $\mathbf{I}$ & $\mathbf{v}$ & I & $\mathbf{V}$ & I \\
\hline Período & 83,5906 & 53,3793 & & & 83,5906 & 53,3793 & 37,02 & 20,67 & 0,0002 & 0,0000 \\
\hline Local & 7,12146 & 0,0 & & & 7,12146 & 0,0 & 2,45 & 0,00 & 0,1201 & 1,0000 \\
\hline Cuidado Parental & 36,3497 & 1,00446 & & & 36,3497 & 1,00446 & 13,68 & 0,34 & 0,0003 & 0,5605 \\
\hline
\end{tabular}

Fonte: Elaborada pelos autores.

A análise de frequência (Tabela 7) evidencia que muitas das respostas dos pescadores a respeito da ecologia reprodutiva das espécies de peixes avaliadas estiveram divergentes das informações disponibilizadas na literatura científica. As etnoespécies traíra (Hoplias spp.), pescada (P. squamosissimus) e tucunaré (C. monoculus) alcançaram frequência menor que $10 \%$ de respostas semelhantes às respostas da literatura em mais de uma das categorias reprodutivas estudadas.

Tabela 7. Análise de frequência das respostas condizentes com a literatura científica sobre ecologia reprodutiva dos principais peixes capturados pelos pescadores da Vila São Cosme e Ilha de Esaú. Em negrito, frequência menor ou igual a 10\% que indica divergência entre respostas científicas e dos pescadores. V: Vila São Cosme; I: Ilha de Esaú.

\begin{tabular}{ccccccc}
\hline \multirow{2}{*}{ ETNOESPÉCIE } & \multicolumn{2}{c}{ Período de desova } & \multicolumn{2}{c}{ REPRODUÇão } \\
& $\mathbf{V}$ & $\mathbf{I}$ & $\mathbf{V}$ & $\mathbf{I}$ & \multicolumn{2}{c}{ Cuidado Parental } \\
& $16,67 \%$ & $15,97 \%$ & $15,83 \%$ & $15,97 \%$ & $13,33 \%$ & $14,58 \%$ \\
\hline Curimatã & $15,83 \%$ & $15,28 \%$ & $15,83 \%$ & $16,67 \%$ & $13,33 \%$ & $13,89 \%$ \\
Piau & $\mathbf{1 , 6 7 \%}$ & $\mathbf{3 , 4 7 \%}$ & $\mathbf{9 , 1 7 \%}$ & $11,81 \%$ & $\mathbf{5 , 8 3 \%}$ & $\mathbf{9 , 0 3 \%}$ \\
Traíra & $13,33 \%$ & $10,42 \%$ & $15 \%$ & $15,28 \%$ & $15 \%$ & $15,97 \%$ \\
Cará-tilápia & $12,50 \%$ & $\mathbf{9 , 7 2 \%}$ & $\mathbf{1 , 6 7 \%}$ & $\mathbf{0 , 6 9 \%}$ & $12,50 \%$ & $\mathbf{7 , 6 4 \%}$ \\
Pescada & $\mathbf{5 , 8 3 \%}$ & $\mathbf{4 , 8 6 \%}$ & $11,67 \%$ & $14,58 \%$ & $\mathbf{9 , 1 7 \%}$ & $16,67 \%$ \\
Tucunaré & & &
\end{tabular}

Fonte: Elaborada pelos autores.

Nestes termos, para o período reprodutivo, estas três espécies obtiveram frequência baixa, conforme apontado pela ANOVA, que indicou significativa diferença entre as médias para esta categoria. Destaque para traíra e tucunaré que obtiveram percentual menor que 6\% nas duas comunidades. Para a literatura, estes peixes desovam no período chuvoso (Marques et al., 
2001; Winemiller, 2001), enquanto a maior parte dos pescadores observou que estes peixes são, frequentemente, encontrados ovados no mesmo período.

É provável que esta observação ocorra pelo fato dos peixes dos gêneros Hoplias e Cichla apresentarem maturação sexual em média três a quatro meses antecedentes ao período de desova (Marques et al., 2001). Com isso, somando-se o período de maturação sexual aos três meses anuais do período chuvoso, totaliza entre seis e sete meses o período que os pescadores podem encontrar estes peixes ovados. Logo, o estado ovado induz a interpretação, para os pescadores, de que o peixe está desovando. Este padrão de interpretação sobre o período reprodutivo de espécies piscívoras foi observado em pescadores do estado do Rio Grande do Norte (Silva et al., 2014). Contudo, ainda que os pescadores não identifiquem ao certo o período de desova destas espécies, por ocorrer no período chuvoso, elas acabam sendo protegidas pelo período de três meses de defeso da piracema (fevereiro a abril), quando é proibida a pesca com usos de malhas.

Quanto ao local de desova da pescada ( $P$. squamosissimus), houve um percentual menor que 2\%, na Vila São Cosme e na Ilha de Esaú, de respostas que concordaram com a literatura científica, considerando que esta espécie desova em água corrente (Camargo \& Lima-Júnior, 2008). Porém, é importante recordar que a ANOVA não apontou diferença significativa entre as médias das espécies para local de reprodução. Contudo, é válido destacar que a maior frequência de respostas dos pescadores é que esta espécie desova em águas profundas dos reservatórios. Provavelmente, por se tratar de uma espécie exótica (Froese \& Pauly, 2021) e que vive em águas profundas (Goulding, 1980), a resposta dos pescadores indicou que estes não conhecem muito a respeito do local de desova da pescada. Todavia, há de se considerar ainda que se trata de uma espécie de grande porte que alcança tamanho máximo superior a cinquenta centímetros (Camargo \& Lima-Júnior, 2008) e que vive em cardumes (Borie et al., 2014). Assim, a migração nos açudes Araras e Edson Queiroz para os afluentes do reservatório durante o período chuvoso, possivelmente, seria vista pelos pescadores. Outra explicação é que os indivíduos desta espécie tenham modificado o hábito reprodutivo nos reservatórios em questão, conforme observado pelos pescadores. Isto porque os afluentes dos açudes Araras e Edson Queiroz possuem intermitência sazonal e pequeno ou médio porte. De fato, esta explicação exige estudo detalhado sobre a ecologia reprodutiva e migratória da pescada em reservatórios do semiárido nordestino.

Em relação ao cuidado parental, os pescadores consideraram que a traíra (Hoplias spp.) não apresenta cuidado parental. A mesma conduta foi considerada para o tucunaré (C. monoculus) na Vila São Cosme. Estas respostas divergiram da literatura científica que considera que estas espécies possuem cuidado parental (Garcia et al., 2019; Winemiller, 2001). Os pescadores justificaram sua resposta com base na agressividade destes peixes, que, por este motivo, não cuidavam de seus filhotes. A propósito, os pescadores relataram canibalismo entre os tucunarés, corroborando com o observado na literatura acadêmica (Novaes et al., 2004).

Para a pescada (P. squamosissimus), a análise de frequência foi de 6,74\% de respostas, na Ilha de Esaú, em acordo com a literatura científica, que afirma que esta espécie não possui cuidado parental (Garcia et al., 2019). O baixo percentual desta análise ocorreu porque em torno de $45 \%$ dos pescadores desta comunidade não souberam responder sobre a existência ou não de cuidado parental nesta espécie.

Tendo em consideração o cará-tilápia ( $O$. niloticus), que apresentou percentual de respostas em correspondência com a literatura superior a $15 \%$ para cuidado parental, foram minuciosos os conhecimentos apresentados pelos pescadores. De acordo com estes trabalhadores, o cará-tilápia guarda seus ovos na boca ou guelras (brânquias), com esse comportamento, protegem os filhotes de predadores até estes atingirem tamanho para sobreviver sozinhos. Ainda, relataram que, quando capturados, estes peixes liberam sua prole na água a fim de que sobrevivam. Esta descrição foi encontrada de forma semelhante na literatura, onde o referido peixe armazena a ova e as larvas na cavidade bucal (Philippart \& Ruwet, 1992).

Observou-se riqueza de conhecimentos sobre a ecologia reprodutiva dos peixes capturados entre os pescadores de açudes do médio rio Acaraú. Prontamente, esses saberes fornecem informações que podem contribuir para a manutenção do 
estoque pesqueiro futuro e a conservação da biodiversidade. Nestes termos, este trabalho poderá contribuir com estudos que objetivem analisar a influência da atividade de pesca no sucesso reprodutivo da ictiofauna, uma vez que aponta elementos da relação entre pescador e natureza.

\section{Conclusão}

As comunidades de pescadores artesanais Ilha de Esaú e Vila São Cosme desempenham a pesca como atividade fundamental para o sustento familiar. Uma vez que esta atividade perpassa a cultura destas populações em vários sentidos, os pescadores locais apresentaram abundantes conhecimentos a respeito da bioecologia dos principais peixes por eles capturados.

Quanto à etnoictiologia, concluiu-se, de forma geral, que os pescadores artesanais dos reservatórios estudados reservatórios possuem conhecimentos consistentes sobre a ecologia geral, trófica e reprodutiva da ictiofauna capturada, vivenciando empiricamente muitas informações presentes na literatura acadêmica. Não obstante, notou-se, pelos resultados estatísticos, que a maior parte das respostas não condizentes com os dados científicos é referente às espécies exóticas à bacia hidrográfica do rio Acaraú. Isto sugere maior conhecimento das espécies nativas, por manejarem-nas há mais tempo e/ou por mudança no comportamento das espécies exóticas ante um ecossistema com características marcadamente divergentes dos ecossistemas de origem destas espécies.

Estima-se que os conhecimentos ecológicos destas populações de pescadores artesanais podem colaborar em futuros estudos científicos, uma vez que aponta lacunas a serem investigadas sobre a ecologia de peixes do semiárido nordestino. Orienta-se que esses saberes e experiências sejam considerados na elaboração de planos de gestão e manejo sustentável dos recursos hídricos e pesqueiros de reservatórios do semiárido nordestino brasileiro.

\section{Agradecimentos}

Os autores agradecem às comunidades Vila São Cosme e Ilha de Esaú pela participação no estudo e pela hospitalidade. Ao Departamento Nacional de Obras Contra as Secas (DNOCS), pela disponibilização de dados e informações sobre os reservatórios estudados. À Fundação Cearense de Apoio ao Desenvolvimento Científico e Tecnológico pelo auxílio (FUNCAP) à esta pesquisa.

\section{Referências}

Abelha, M. C. F., Agostinho, A. A. \& Goulart, E. (2001). Plasticidade trófica em peixes de água doce. Acta Scientiarum, 23, 425-434. 10.4025/actascibiolsci.v23i0.2696

Albuquerque, U. P., Lucena, R. F. P. \& Alencar, N. L. (2010). Métodos e técnicas para coleta de dados etnobiológicos. In: Albuquerque, U. P., Lucena, R. F. P. \& Cunha, L. V. F. C. (Org.s) Métodos e técnicas na pesquisa etnobiológica e etnoecológica. NUPEEA, Recife.

Bwanika, G. N., Chapman, L. J., Kizito, Y. \& Balirwa, J. (2006). Cascading effects of introduced Nile perch (Lates niloticus) on the foraging ecology of Nile tilapia (Oreochromis niloticus). Ecology of freshwater fish, 15 (4), 470-481. 10.1111/j.1600-0633.2006.00185.x

Begossi, A., Salivonchyk, S., Lopes, P. F. M. \& Silvano, R. A. M. (2016). Fishers' knowledge on the coast of Brazil. Journal of Ethnobiology and Ethnomedicine, 12 (20), 1-34. 10.1186/s13002-016-0091-1

Borie, A., Mok, H. K., Chao, N. L. \& Fine, M. L. (2014). Spatiotemporal Variability and Sound Characterization in Silver Croaker Plagioscion squamosissimus (Sciaenidae) in the Central Amazon. PLoS ONE, 9 (8), e99326. 10.1371/journal.pone.0099326

Camargo, M. \& Lima-Júnior, W. M. A. (2008). Aspectos da biologia reprodutiva de seis espécies de peixes de importância comercial do médio rio Xingu bases para seu manejo. Scientific Magazine Uakari, 3 (1), 64-77. Disponível em link: https://uakari.emnuvens.com.br/UAKARI/article/view/20

Capra, L. G. \& Bennemann, S. T. (2009). Low feeding overlap between Plagioscion squamosissimus (Heckel, 1840) and Cichla monoculus (Spix, Agassiz, 1831), fishes introduced in tropical reservoir of South Brazil. Acta Limnologica Brasiliensia, 21 (3), 343-348. https://www.ablimno.org.br/acta/pdf/v21n3a210307.pdf

Casatti, L., Langeani, F. \& Castro, R. M. C. (2001). Peixes de riacho do parque estadual Morro do Diabo, bacia do alto rio Paraná, SP. Biota NeotropicaI, 1 (1), 1-15. 10.1590/S1676-06032001000100005 
Costa, I. D. \& Angelini, R. (2020). Gut content analysis confirms the feeding plasticity of a generalist fish species in a tropical river. Acta Limnologica Brasiliensia, 32 (1), e21. 10.1590/S2179-975X7819

Diegues, A. C. (2014). The role of ethnoscience in the build-up of ethnoconservation as a new approach to nature conservation in the tropics. The case of Brazil. Revue d'ethnoécologie, 6 (1), 132-146. 10.4000/ethnoecologie.1956

Dill, L. M. (1983). Adaptative flexibility in the foraging behavior of fishes. Canadian Journal of Fisheries and Aquatic Sciences, 40 (4), 398-408. $10.1139 /$ /f83-058

Durães, R., Pompeu, P. S. \& Godinho, A. L. (2001). Alimentação de quatro espécies de Leporinus (Characiformes, Anostomidae) durante a formação de um reservatório no Sudeste do Brasil. Iheringia (Série Zoologia), (90), 183-191. 10.1590/S0073-47212001000100019

Froese, R. \& Pauly, D. (Eds). (2021). FishBase. World Wide Web Electronic Publication, versão 02/2011. www.fishbase.org (verificado em 19 de junho de 2021).

Fugi, R., Hahn, N. S. \& Agostinho, A. A. (1996). Feeding styles of five species of bottomfeeding fishes of the high Paraná River. Environmental Biology of Fishes, 46 (3), 297-307. 10.1007/BF00005006

Garcia, D. A. Z., Vidotto-Magnoni, A. P., Costa, A. D. A., Casimiro, A. C. R., Jarduli, L. R., Ferraz, J. D., Almeida, F. S. \& Orsi, M. L. (2019). Importance of the Congonhas River for the conservation of the fish fauna of the Upper Paraná basin, Brazil. Biodiversitas Journal of Biological Diversity, 20 (2), 474-481. $10.13057 /$ biodiv/d200225

Gonçalves, C. S., Braga, F.M. S. \& Casatti, L. (2018) Trophic structure of coastal freshwater stream fishes from an Atlantic rainforest: evidence of the importance of protected and forest-covered areas to fish diet. Environmental Biology of Fishes, 101 (6), 933-948. 10.1007/s10641-018-0749-8

Goulding, M. (1980). The fishes and the forest: Explorations in Amazonian Natural History. Berkeley. University of California Press.

Gubiani, E. A., Ruaro, R, Ribeiro, V. R., Eichelberger, A. C. A., Bogoni, R. F., Lira, A. D., Cavalli, D., Piana, P. A. \& Weferson, J. G. (2018). Non-native fish species in Neotropical freshwaters: how did they arrive, and where did they come from?. Hydrobiologia, 817 (1), 57-69.

Hahn, N. S., Loureiro, V. E. \& Delariva, R. L. (1999). Atividade alimentar da curvina Plagioscion squamosissimus (Heckel, 1840) (Perciformes, Sciaenidae) no rio Paraná. Acta Scientiarum, 21 (2), 309-314. 10.4025/actascibiolsci.v21i0.4438

Ladislau, D. S., Souza, P. L., Aride, P. H. R., Oliveira, A. T. \& Gubiani, E. A. (2021). Current situation and future perspectives of ethnoichthyology in Brazil. Ethnobiology and Conservation, 10 (9), 1-35.

Lowe-Mcconnell, R. H. (1999). A fauna de peixes neotropical. In: Lowe-Mcconnell, R. H. (Org.) Estudos ecológicos de comunidades de peixes tropicais. EDUSP, São Paulo.

Marques, D. K. S., Gurgel, H. C. B. \& Lucena, I. (2001). Época de reprodução de Hoplias malabaricus Bloch, 1794, (Osteichthyes, Erythrinidae) da barragem do rio Gramame, Alhandra, Paraíba, Brasil. Revista Brasileira de Zoociências, 3 (1), 61-67.

Moura, C. D. C. F. L., Oliveira, J. F., Novaes, J. L. C., Costa, R. S. D., Araújo, D. D. A. D. \& Peretti, D. (2018). O impacto de um experimento de biomanipulação na dieta da ictiofauna de um reservatório do semiárido neotropical. Acta Limnologica Brasiliensia, 30 (1), e107.

Odum, E. P. \& Barrett, G. W. (2008) Fundamentos de Ecologia. Cengage Learning, São Paulo.

Novaes, J. L. C., Caramaschi, E. P. \& Winemiller, K. O. (2004). Feeding of Cichla monoculus Spix, 1829 (Teleostei: Cichlidae) during and after reservoir formation in the Tocantins River, Central Brazil. Acta Limnologica Brasiliensia, $16 \quad$ (1), 41-49. https://www.ablimno.org.br/acta/pdf/acta_limnologica_contents1601E_files/art4_16(1).pdf

PAST: Paleontological Statistics Software Package for Education and Data Analysis. (2001). Versão $4 . \quad$ https://palaeoelectronica.org/2001_1/past/issue1_01.htm. Paleontologica Electronic. (2019). ver 22(3). https://palaeo-electronica.org/

Pereira, B. E., A. C. Diegues. 2010. Conhecimento de populações tradicionais como possibilidade de conservação da natureza: uma reflexão sobre a perspectiva da etnoconservação. Desenvolvimento e Meio ambiente, 22 (22): 37-50. 10.5380/dma.v22i0.16054

Philippart, J. C. \& Ruwet, J. C. (1982). Ecology and distribution of tilapias. In: Pullin, R. S. V. \& Lowe-Mcconnell, R. H. (Org.s) The biology and culture of tilapias. ICLARM - International Center for Living Aquatic Resources Management, Manila.

Pompeu, P. S. \& Godinho, A. L. (2001). Mudança na dieta da traíra Hoplias malabaricus (Bloch) (Erythrinidae, Characiformes) em lagoas da bacia do rio Doce devido à introdução de peixes piscívoros. Revista Brasileira de Zoologia, 18 (4), 1219-1225.

Prado, H. M., \& Murrieta, R. S. S. (2015). Ethnoecology in perspective: the origins, interfaces and current trends of a growing field. Ambiente \& Sociedade, 18 (4), 139-160. 10.1590/1809-4422ASOC986V1842015

Pratiwy, F. M. \& Kohbara, J. (2018). Dualistic feeding pattern of Nile tilapia (Oreochromis niloticus, L.) reared under different self-feeding system conditions. Aquaculture Research, 49 (2), 969-976. 10.1111/are.13544

Rabelo, H. \& Araujo-Lima, C. A. R. M. (2002). A dieta e o consumo diário de alimento de Cichla monoculus na Amazônia Central. Acta Amazônica, 32 (4), 707-724. 10.1590/1809-43922002324724

Resende, E. K. (1992). Bioecologia do curimatã, Prochilodus lineatus no Pantanal do Miranda Aquidauana, Mato Grosso do Sul, Brasil. Acta Limnologica Brasiliensia, 4 (1), 261-276.

Sánchez-Botero, J. I. \& Araujo-Lima, C. A. R. M. (2001). As macrófitas aquáticas como berçário para a ictiofauna da Várzea do rio Amazonas. Acta Amazônica 31 (3), 437-447. 10.1590/1809-43922001313447 
Research, Society and Development, v. 11, n. 2, e44711225733, 2022

(CC BY 4.0) | ISSN 2525-3409 | DOI: http://dx.doi.org/10.33448/rsd-v11i2.25733

Silva, E. F., Oliveira, J. E. L. \& Schiavetti, A. (2014). Conhecimento Ecológico Local (CEL) na pesca artesanal da Reserva de Desenvolvimento Sustentável Estadual Ponta do Tubarão-RN, Brasil. Boletim do Instituto de $\quad$ Pesca, $40 \quad$ (3), $355-375$. https://www.pesca.agricultura.sp.gov.br/boletim/index.php/bip/article/view/1046

Winemiller, K. O. (1989). Patterns of variation in life history among South American fishes in seasonal environment. Oecologia, 81 (2), $225-241$. 10.1007/BF00379810

Winemiller, K. O. (2001). Ecology of peacock cichlids (Cichla ssp.) in Venezuela. Journal of Aquariculture and Aquatic Sciences, 9, 93-112. 10.1007/BF00379810

Wieczorkowki, J. R. S., Pesovento, A. \& Téchio, K. H. (2019). Etnociência: um breve levantamento da produção acadêmica de discentes indígenas do curso de educação intercultural. Revista Ciências \& Ideias, 9 (3), 153-168. 10.22407/2018.v9i3.948 